\title{
Capítulo 2 Percepción de violencia de género en estudiantes universitarios
}

\section{Chapter 2 Perception of gender violence in university' students}

ALFARO-CÁZARES, Neydi Gabriela†๋*, HABIB-MIRELES, Lizbeth, ZAMBRANO-GARZA, Mónica y RODRÍGUEZ-LUIS, Osvelia Esmeralda

Universidad Autónoma de Nuevo León. Facultad de Ingeniería Mecánica y Eléctrica

Universidad Autónoma de Nuevo León. Facultad de Odontología

ID $1^{\mathrm{er}}$ Autor: Neydi Gabriela, Alfaro-Cázares / ORC ID: 0000-0002-2618-1634

ID $1^{\text {er }}$ Coautor: Lizbeth, Habib-Mireles / ORC ID: 0000-0003-2604-3861, Researcher ID Thomson: AAL-3798-2020, CVU CONACYT ID: 481593

ID 2 ${ }^{\text {do }}$ Coautor: Mónica, Zambrano-Garza / ORC ID: 0000-0002-0918-6628, CVU CONACYT ID: 629257

ID $3^{\text {er }}$ Coautor: Osvelia Esmeralda, Rodríguez-Luis / ORC ID: 0000-0002-5088-9851, CVU CONACYT ID: 391708

DOI: $10.35429 / \mathrm{H} .2020 .6 .15 .26$

N. Alfaro, L. Habib, M. Zambrano y O. Rodríguez

neydi.alfarocr@uanl.edu.mx

A. Marroquín, J. Olivares, L. Cruz y A. Bautista. (Coord) Sociedad, violencia de género. Handbooks-CECORFAN-Mexico, Querétaro, 2020. 


\section{Resumen}

Este capítulo se enfoca en un análisis de investigación sobre la violencia a la que están expuestos ambos géneros, el objetivo es conocer cuál es el género que mayor maltrato recibe acotándolo a estudiantes de ingeniería, y cuál es el tipo de violencia que sufre, si es verbal, física o de bullying. La metodología utilizada para este trabajo fue de enfoque cuantitativo transversal, la primera fase de la investigación fue de modo exploratorio realizando una revisión bibliográfica en revistas y documentos que tuvieran relación con género, abuso, escuela y que estos fueran de actualidad y en la segunda fase se contó con la técnica de una encuesta en línea, la cual, se estructuró con opción múltiple, aplicándola a través de la plataforma Google Forms, para demostrar la hipótesis de que los estudiantes de ambos géneros son sujetos de algún tipo de maltrato, se obtuvo una muestra compuesta por 229 estudiantes con edades comprendidas entre los 17 y 28 años, con una edad promedio de 19.8, son alumnos de nivel superior y que están cursando una carrera de ingeniería de los semestres de sexto a octavo, el escenario es una universidad pública en el noreste del país, los cuales participaron mediante la técnica de la aplicación de la encuesta de forma anónima, y dado que se ha demostrado en investigaciones previas que el género femenino es más susceptible de ser violentado que el masculino. Los resultados de la investigación muestran que el sexo masculino, si bien, no está exento de sufrir abuso, sigue siendo el género femenino el más vulnerable de recibir algún tipo de violencia.

\section{Violencia, estudiante universitario, género}

\section{Abstract}

This investigation focuses on a research analysis on violence to which both genders are exposed, the objective is to know which is the gender that receives the most abuse, limiting it to engineering students, and what is the type of violence suffered, if it is verbal, physical or bullying. The methodology used for this work was of a cross-sectional quantitative approach, the first phase of the investigation was exploratory, conducting a bibliographic review in journals and documents that were related to gender, abuse, school looking for them to be recent, and in the second phase an online survey, which was structured with multiple choice items, applying it through the Google Forms platform, to demonstrate the hypothesis that students of both genders are subject to some type of abuse. The survey was applied to 229 engineering students ages 17 and 28 years old, with an average age of 19.8, from the sixth to eighth semesters in their careers at a public university in the northeast of the country, which participated anonymously. And as it's been demonstrated in previous analysis statements such as those that indicate that the female gender is more susceptible to being abused than the male. The results of the research show that, although the male sex is not exempt from suffering abuse, is still the female gender the most vulnerable to receiving some type of violence.

\section{Violence, higher education students, gender}

\section{Introducción}

La Organización de las Naciones Unidas para la Educación, la Ciencia y la Cultura (UNESCO, 2019) reporta que existen 246 millones de adolescentes y niñas que pueden ser víctimas de violencia, al interior y alrededor de las escuelas, por no adaptarse a las normas sexuales y de género predominantes. Hoy en nuestros días, ocurre con mayor frecuencia algún tipo de abuso, y probablemente esto no sea nada nuevo, sino que actualmente se tiene mayor acceso a redes sociales y esta información se da a conocer, incluso se puede considerar que comienza a existir la conciencia de la denuncia, otro aspecto a tomar en cuenta, es que quizás la sociedad en su conjunto, y el individuo en lo particular, tienen mayor conciencia de que existen diferentes formas de ser violentado y que esto sucede cuando se ejecutan acciones en contra de su voluntad (Buvinic, 2005), sin embargo, la violencia no es exclusiva de un país, pueblo o lugar porque se encuentra en cualquier parte del mundo, las investigaciones se han enfocado en identificar cuál es el tipo de abuso que el género femenino recibe, olvidando o dejando de considerar que también el género masculino es igualmente susceptible de ser violentado, con sus considerables diferencias. 
Actualmente el universo está comprendido por un mayor número de jóvenes, ya que representan alrededor de un $40 \%$ de la población mundial y en México un aproximado al $43 \%$ de los habitantes tiene menos de 29 años de edad, es decir, jóvenes. El país ha presentado de manera creciente situaciones de violencia, existiendo una realidad social de abuso en la que viven los jóvenes hoy en día, incluyendo a los universitarios. La UNESCO (2019) realizó un estudio sobre la violencia y bullying en las escuelas, donde quedó demostrado que uno de cada tres estudiantes fue intimidado en la mayor parte de los continentes, excepto Europa y América del Norte, donde se vive más el acoso físico en el género masculino porque son más violentados físicamente en comparación con las mujeres, por esta razón, se consideró importante realizar una investigación a través de una encuesta para analizar cuál es el género que está más susceptible a ser violentado en una universidad pública, y cuál es el tipo de abuso que más reciben, se sometió a comprobación si la violencia se presenta solo en el género femenino, como establecen la mayoría de los documentos consultados a nivel internacional, nacional y local, o si no se está considerando en los estudios al género masculino, y hasta cierto punto, dejándolo indefenso por falta de evidencias sobre la incidencia a ser violentado, como señala Bueno, M. (2010) en: "Hombres maltratados, una realidad silenciada", donde señala que también existe el maltrato en el género masculino, pero que los datos no son exhibidos, probablemente por la cultura en donde se desenvuelven.

\subsection{Objetivo}

Proporcionar información sobre la percepción de violencia de género en estudiantes universitarios.

\subsection{Hipótesis}

Los estudiantes universitarios de género masculino son sujetos de algún tipo de violencia, al igual que las mujeres.

Esta investigación tiene como objetivo obtener información sobre la percepción de violencia en estudiantes de nivel superior, analizando cuáles son los tipos de abusos que reciben, también conocer si lo reciben de su mismo género o del género contrario, y entonces poder esgrimir recomendaciones y proponerlas para que disminuya la violencia en la institución, el estudio se enfocó en una escuela de ingeniería, en una universidad pública que cuenta en su matrícula con una mayoría de estudiantes de género masculino, con la finalidad de demostrar la hipótesis de que los estudiantes de ambos géneros son sujetos de algún tipo de abuso, se optó que este trabajo se realizara a través del método cuantitativo que la primera fase de la investigación fue exploratorio realizando una revisión bibliográfica en revistas y documentos que tuvieran relación con género, violencia, escuela y que estos fueran de actualidad y en la segunda fase, la aplicación de una encuesta en línea a través de la plataforma Google Forms, que se estructuró con opción múltiple, teniendo como sujetos de estudio a alumnos de sexto a octavo semestre, la muestra estuvo compuesta por un total de 229 estudiantes de ingeniería en una escuela pública, con edades comprendidas entre los 17 y 28 años, con una edad promedio de 19.8 y una desviación estándar de 1.56 .

\subsection{Desarrollo}

En este apartado se abordarán la definición de violencia, características y algunos estudios realizados por autores y por organismos como la Organización Mundial de la Salud (OMS), la UNESCO y el Instituto Nacional de Estadística y Geografía (INEGI). La OMS (2018) define género como: “el concepto social de las funciones, comportamientos, actividades y atributos que cada sociedad considera apropiados para los hombres y las mujeres", quedando de manifiesto que esta conceptualización contempla ambos géneros.

En cuanto a violencia, la OMS la establece como:

"El uso deliberado de la fuerza física o el poder, ya sea en grado de amenaza o efectivo, contra uno mismo, otra persona o un grupo o comunidad, que cause o tenga muchas probabilidades de causar lesiones, muerte, daños psicológicos, trastornos del desarrollo o privaciones” (OPS-OMS, 2002, pág. 3) 
Por su parte Dobarro (2014) clasifica los tipos la violencia que se viven dentro de la escuela como física, verbal, de exclusión social, disrupción en el aula, violencia a través de las TIC y del profesor hacia el alumno, mientras que Buvinic (2005), la define como el acto de ejecutar acciones físicas o psicológicas en contra de la voluntad de la persona.

Para el autor Sanmartín (2007), la violencia es clasificada como se muestra en la figura 2.1, que establece que género y sexo son diferentes y que la violencia que sufre el género femenino es porque no cumplen con el rol que le corresponde en la sociedad y esta se puede dar en el trabajo, escuela, casa, pantallas, tradiciones culturales, por otro lado se encuentra la violencia en la escuela, esta se puede dar entre profesores y alumnos, alumno y alumno que va desde una pelea, insultos y exclusión, haciendo uso de un poder sobre otro. Se establece que en ocasiones es tan fuerte el acoso, que puede la víctima tener repercusiones psicológicas, la figura 2.1 muestra las diferentes clasificaciones de violencia, centrándonos para esta investigación en el escenario de la universidad, y conocer cuál era la forma a través de la cual los estudiantes universitarios reciben violencia, para este estudio se acotó la violencia de acuerdo al escenario, por ser el centro de estudio una institución educativa y las relaciones dentro de la misma.

Figura 2.1 Clasificación de la violencia de acuerdo a Sanmartín (2007)

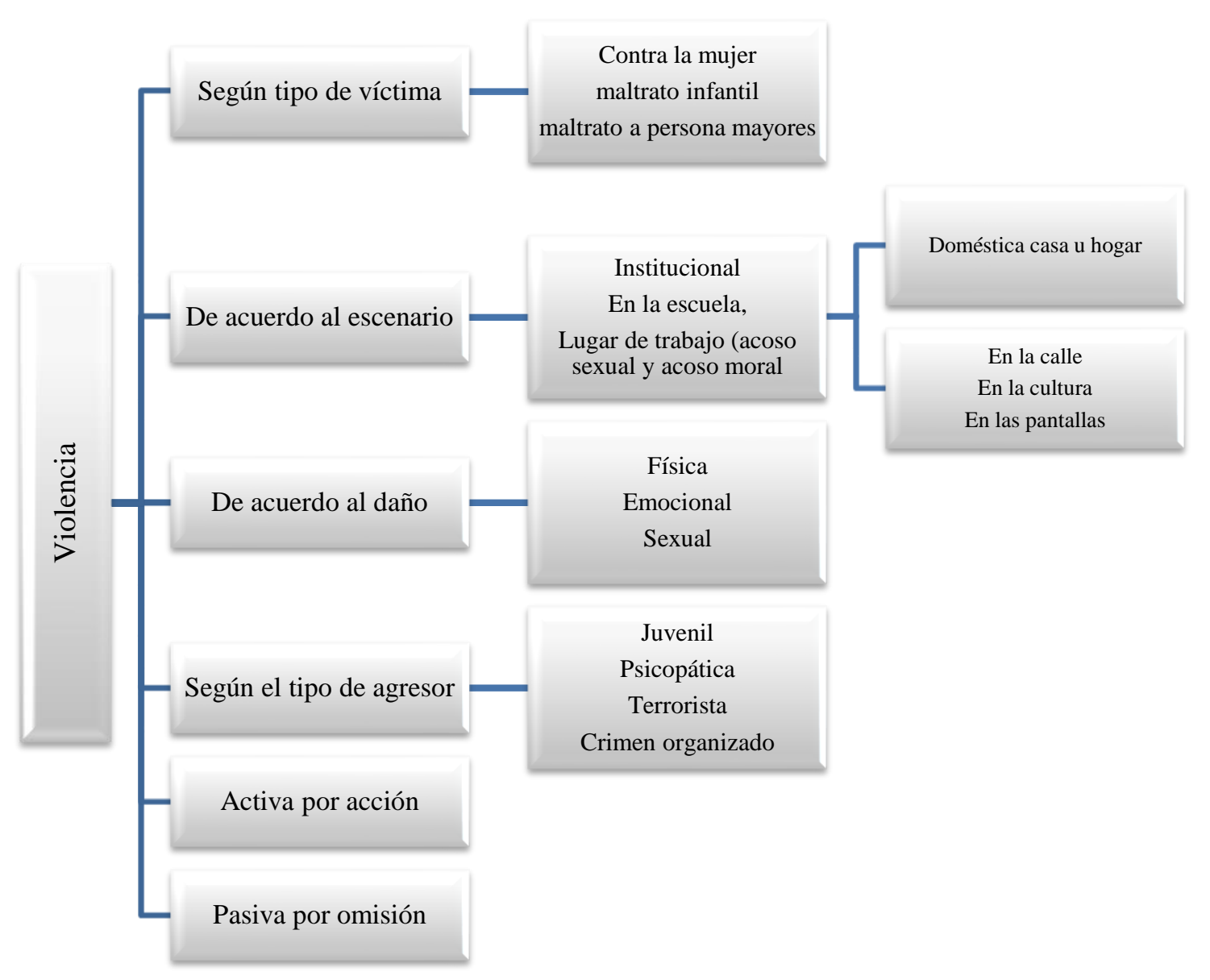

Fuente: Elaboración propia en base Sanmartín (2007)

La conducta violenta para otros autores puede ser directa por ejemplo mediante insultos, patadas, empujones, etc., pero también existe la conducta indirecta que se lleva a cabo mediante la exclusión social, los chismes o los falsos rumores, de boca en boca o mediante el uso de redes sociales. Es común que la conducta violenta sea repetitiva porque las personas victimadas por temor suelen no reaccionar. (Mateo, Mesas y Ruiz, 2015).

Guedes, García-Moreno y Colombini (2017), mencionan que en muchas ocasiones las conductas violentas se dejan pasar porque existe cierta tolerancia dentro de las normas sociales, que impiden que las víctimas pidan ayuda, una de las formas más comunes para obtener información en cuestión de violencia que ha sido mayormente utilizada es a partir de cuestionarios, en donde se les pide a las personas contestar de manera honesta para poder conocer con qué frecuencia han sido victimados de manera verbal, física, emocional, pero la mayoría de los estudios está centrada en el género femenino, o bien en menores de edad. 
Por su parte en México se ha creado la Ley General de Acceso de las Mujeres a una Vida Libre de Violencia $(2007$, p.2) artículo 5, fracción IV que establece violencia como “...cualquier acción u omisión, basada en su género, que les cause daño o sufrimiento psicológico, físico, patrimonial, económico, sexual o la muerte tanto en el ámbito privado como en el público.”

Por su parte la UNESCO considera la violencia y el acoso escolar como un problema mundial, y esto quedó demostrado a través de un estudio que realizó en 144 países donde los resultados dejaron de manifiesto que uno de cada tres estudiantes que representaron el 32\% de la muestra total había sido intimidado por otros alumnos, que la violencia física la reciben más los niños y está presente en diferentes regiones excepto Norteamérica y Europa donde la violencia psicológica, es la más susceptible de presentarse en niñas, y donde el acoso sexual es el segundo tipo de violencia con mayor ocurrencia, de una u otra forma la violencia escolar se presenta en ambos géneros, también reciben acoso en línea y a través de dispositivos móviles. Otro tipo de violencia que sufren los estudiantes es por discriminación derivada de diferencias en su apariencia física, raza, nacionalidad y color de piel. (UNESCO, 2019)

Actualmente en su mayoría los análisis se enfocan solo en la violencia que sufre el género femenino, encontrando estadísticas en México dirigidas a mujeres y no habiendo encontrado información sobre violencia hacia el género masculino, probablemente no se considere una prioridad para la sociedad o quizás tiene relación con cuestiones culturales, este trabajo aborda la violencia de género, el cual en ocasiones es pasado por alto.

México, de acuerdo al Índice global de Paz (2018), se encuentra en el lugar 62 de 163 países con menor paz y Nuevo León ocupa el décimo noveno lugar de acuerdo al INEGI (2018) es una problemática que se ha incrementado en los últimos meses, y los reportes muestran que la violencia no es exclusiva de género, en la actualidad se ha considerado que la población sujeta a violencia es la mujer; sin embargo, también existe la violencia hacia los hombres, aún y cuando no es común que ellos externen ser víctimas de maltrato, por el entorno cultural, como lo menciona Giraldo (1972) que:

"Un verdadero macho no puede tolerar que su mujer le pegue o ni siquiera que no le obedezca. Un hombre debe aparecer como el jefe de la casa ante sus amigos hombres si no ha de perder su fama de macho", p. 298

Y esto sucede porque no es común que el hombre sea el que exponga que vive alguna forma de violencia, ya que aceptar que es violentado puede ocasionar un reproche social o por lo menos quedar exhibido, por el rol cultural que juega en la sociedad, donde el hombre y mujer desempeñan diferentes papeles de acuerdo con el sexo con que se nace y están sujetos a determinados derechos y obligaciones. Queda de manifiesto que la violencia puede ser recibida o ejercida de igual forma en ambos sexos, solo que en las investigaciones consultadas por las autoras se encontró que esta se concentra en la violencia recibida por el rol femenino, dejando aún lado que el hombre también puede ser sujeto de recibir violencia ya sea emocional, física y de salud, autoinfligida, interpersonal y colectiva, en el contexto educativo.

Sin embargo, durante el 2020 el INEGI (2019), mostró que los resultados de su encuesta reportan que las mujeres jóvenes con edades entre 20 y 44 años son más expuestas a los abusos y a la violencia de la pareja o de cualquier otro agresor, y que 76 de cada 100 mujeres en el rango de edad entre 30 a 44 años de edad, han enfrentado al menos un episodio de violencia o abuso. La violencia sexual se ve representada mayormente en mujeres jóvenes entre 18 y 29 años, donde la mitad ha sido agredida sexualmente, por otra parte, las niñas de 15 a 17 años han sido víctimas de niveles de violencia sexual, emocional y física y a su corta edad, ya han sido víctimas de abusos de diversa índole.

Se considera que aquel individuo que comete actos de violencia puede ser por las consecuencias directas que se viven en el hogar, que, en el caso de los varones, ocurre porque han presenciado diferentes tipos de violencia y que pueden ser violentos con sus compañeras e incluso hijos al llegar a la edad adulta según Águila, Hernández y Hernández (2016). 


\subsection{Metodología}

En una primera fase de la investigación en modo exploratorio, se realizó una revisión bibliográfica localizando un estudio que realizó Safranoff (2017) para analizar y obtener indicadores de maltrato es el declarando verbalmente por la mujer encuestada que ha sufrido violencia física, sexual o psicológica y que lo ha recibido durante el último año, también se consideró el maltrato técnico que se presenta en situaciones actuales que estaban sufriendo, el estudio comprendió una muestra de 5450 mujeres, mayores de 18 años, residentes de España durante el año 2011, que tuvieran una relación con duración arriba de un año, se les aplicó una encuesta analizando los resultados mediante tablas de contingencia, considerando las variables que fueron dicotomizadas, mostrando que un $45.8 \%$ de mujeres sufren violencia física y tienen conciencia, pero el $54.2 \%$ no tienen conciencia de que reciben maltrato, estableciendo que las mujeres más educadas son más sensibles a identificar cuando son maltratadas, obteniendo de este estudio como conclusiones que el maltrato declarado capta cuando es experimentado en forma física (es la más reconocida), psicológica y sexual (queda excluida), que el género femenino menos educado y de mayor edad tiene dificultades para reconocer que es maltratado.

Por otra parte, Barredo (2017) realizó un estudio de carácter exploratorio sobre la violencia de género en estudiantes universitarios en Ecuador, aplicando una encuesta que estuvo compuesta por un testeo teórico y empírico aplicado a 2569 estudiantes compuestos por 1468 mujeres y 1101 hombres considerando la edad en su mayoría hasta 34 años, esta encuesta se analizó a través de un procedimiento estadístico descriptivo mediante una matriz de datos en Excel 2010, y posteriormente explorados a través de IBM SPSS Statistics versión 20.0, obteniendo que cinco de cada diez mujeres recibían violencia de género, dos de cada diez estudiantes aseguraban que ejercieron alguna vez violencia contra su pareja, por lo que se concluye que la violencia de género en este estudio al igual que el anterior está estrechamente relacionado con factor económico, social y que es una violencia que está declarada porque están conscientes de ella.

Durante el 2020, Latorre, Tena, Murillo y Toscano, realizaron un estudio con el objetivo de analizar la relación entre rendimiento académico en estudiantes universitarios y su relación con la percepción del clima escolar, este fue aplicado a 333 estudiantes de la facultad de Psicología de una universidad pública en Michoacán, que cursaban entre 3ro., 5to., 7mo. y 9no. semestre con una edad entre 18 y 29 años compuesta por 259 mujeres y 73 hombres, utilizando un instrumento con opción múltiple para evaluar clima laboral, logros académicos, motivación e interés académico, involucramiento en deportes, servicio comunitario y artes, la cual se analizó a través de una investigación cuantitativa, transversal y analítica de evaluación única utilizando el programa SPSS versión 20.0 aplicando estadística descriptiva, obteniendo como resultado que los estudiantes tienen una percepción del clima escolar regular y consideran que es positiva la relación con docentes y actividades planeadas, pero de forma negativa la convivencia con estudiantes que no se sienten satisfechos con el contexto escolar y tienen un bajo desempeño académico, considerando que la falta de compromiso de estos estudiantes les puede afectar a su promedio al tener que trabajar con ellos.

Por su parte Orellana, Gallardo y Alemani (2020), realizaron una investigación cuantitativa de diseño ex post facto de tipo correlacional a una población de 615 estudiantes de la Universidad de Granada del campus de Melilla a través de un muestreo no probabilístico intencional, que tenía como objetivo conocer cómo influyen las creencias sobre el género de los participantes considerando también las variables de sociodemográficas, el grado de actitud sexista y la relación entre el estereotipo, el instrumento utilizado fueron varios cuestionarios aplicados a través de un código QR: creencias del alumnado, inventario de sexismo ambivalente y uno de carácter sociodemográfico, el análisis se hizo a través de IBM SPSS versión 24.0 para Mac. Obteniendo como resultado que en cuanto estereotipos y actitud sexista es el hombre de origen bereber, que profesa la religión islámica y es muy practicante de la misma, mientras que la mujer no posee tanta carga de estereotipo de género ni actitud sexista, pero que no es creyente y es de origen europeo.

En la segunda fase la aplicación de encuesta en línea, la cual se procesó en base a Dobarro, García, y Núñez (2014), los cuales presentan un análisis de instrumentos que han sido utilizados por investigadores entre los que destacan:

- Autoinformes como: el CEVEO (Díaz-Aguado, Martínez y Martín, 2004), el CUVECO (Fernández-Baena et al., 2011) y el EBIPQ (Del Rey, Elipe, \& Ortega, 2012). 
- Informes institucionales como: Defensor del Pueblo-UNICEF (2007) y del Observatorio Estatal de la Convivencia Escolar (2012).

- Hetereoinformes como: el PKBS-2 (Merrell, 2002), las Parent Rating Scales (PRS) del BASC-2 (Reynolds \& Kamphaus, 2004), el CPCE-P (Peralta, Sánchez, De la Fuente, \& Trianes, 2007) y el Heteroinforme-P del INSEBULL (Avilés \& Elices, 2007).

- Violencia escolar: Cuestionario sobre Intimidación y Maltrato entre Iguales (Ortega y MoraMerchán, 2000).

A partir de estas consultas se realizó el diseño de la encuesta, que estuvo conformada por 18 reactivos, se buscó una aplicación uniforme, que nos permitiera lograr la tasa de respuesta planeada considerando que, por ser temas de violencia, una encuesta anónima y en línea permitiría que los contestaran con mayor honestidad, posteriormente se aplicó la prueba piloto, para conocer si los estudiantes universitarios comprendían en su totalidad cada uno de los ítems. Se realizaron correcciones y eliminación de los que no eran comprendidos.

Con la información antes mencionada, se elaboró un instrumento para identificar la violencia de género en una institución educativa, considerada en su mayoría masculina, por su alto índice de población de diferencia entre hombres que están representados por un 80 a $20 \%$ de mujeres, el cual quedó conformado finalmente por reactivos, que midieron las variables en una escala ordinal y nominal, mismo que fue validado a juicio de expertos. Se consideró que una de las formas más comunes para obtener información en cuestión de violencia y que ha sido utilizada mediante el empleo de cuestionarios anónimos, en los cuales se les solicita a los encuestados contestar de manera honesta para poder conocer con qué frecuencia han sido victimados de manera verbal, física o emocional, siendo esta estrategia metodológica empleada en este trabajo. La aplicación de encuesta en línea, estructurada en opción múltiple a estudiantes de una escuela de ingeniería pública en el norte de México, $(n=229)$, con edades comprendidas entre los 17 y 28 años, con una edad promedio de 19.8 y una desviación estandar de 1.56. En concretofinal esta constituida por el 73,4\% es de género masculino, mientras que el 26,4\% femenino. Otro de los factores considerados en la presente investigación es que el 16,2\% son foráneos por lo que no viven con su familia. Los resultados fueron analizados, realizándose una estadística descriptiva.

\subsection{Resultados}

Del instrumento aplicado se pudo identificar que 168 de los estudiantes encuestados fueron del género masculino y 61 del género femenino, para analizar cuál era la percepción sobre la violencia en ambos géneros, identificando los siguientes resultados:

El primer ítem analizó quiénes se sienten victimados, al no hacer lo que los demás quieren que hagan, obteniendo que el $18 \%$ de los estudiantes encuestados, sí sentían presión y actuaban en múltiples ocasiones en consecuencia a la presión social y en contra de su decisión, este porcentaje en el género masculino fue de $13 \%$, con lo que se demuestra que en este aspecto, los jóvenes son más presionados a realizar determinadas acciones demostrando que hay una diferencia de más de 5\% en la presión social hacia la mujer, siendo esta menos presionada socialmente para actuar en cierta forma.

El segundo ítem, identificó el porcentaje de estudiantes que sentían haber sido objeto de violencia verbal, arrojado los resultados que se muestran en el gráfico 2.1 , donde el $5 \%$ de ambos géneros, expresaron ser víctimas de recibir violencia verbal, con una frecuencia de al menos, una vez a la semana obteniendo que el $31 \%$ de las mujeres y el $40 \%$ de los hombres manifestó que pocas veces, lo que muestra que los hombres son más susceptibles de sufrir mayor violencia verbal que las mujeres de acuerdo a los resultados obtenidos en el estudio. 
Gráfico 2.1 Alumnos que manifestaron ser objeto de violencia verbal y la frecuencia, $(n=229)$

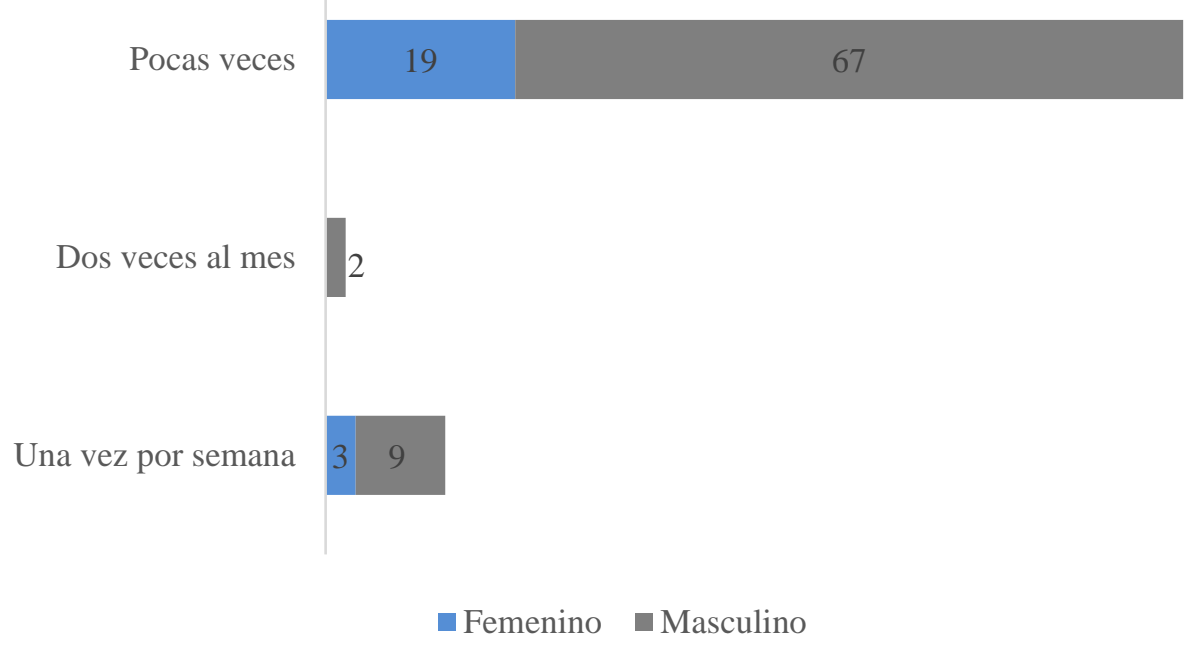

Fuente: Elaboración propia con los datos obtenidos del procesamiento de las respuestas del instrumento

El tercer ítem del instrumento determinó, que los estudiantes se sentían víctimas de rumores, chismes, falsos sobre su persona, los resultados a este cuestionamiento fueron que el $34 \%$ de las mujeres y el $26 \%$ de los hombres habían sido víctimas con poca frecuencia, mientras que el $4 \%$ de las mujeres y $1 \%$ de los hombres esto les sucedía a menudo, con lo que se lo demuestra que en este rubro las mujeres siguen siendo un blanco más fácil de violentar de forma verbal con falsos rumores como lo establece (Mateo, Mesas y Ruiz, 2015).

El cuarto ítem se les solicitaba que mencionaran, con qué frecuencia habían sido victimados físicamente por ejemplo mediante golpes, empujones, etc. Estos resultados se pueden observar en el gráfico 2.2, donde podemos ver que los hombres son los que sufren en mayor frecuencia de violencia física alcanzando un $29 \%$ y las mujeres un $25 \%$ pocas veces, esto coincide al aumentar la frecuencia y $3 \%$ de los hombres fueron victimados al menos dos veces al mes mientras que las mujeres encuestadas el $2 \%$, este resultado coincide con lo que publicó la UNESCO (2019), con la diferencia que este tipo de violencia era más frecuente en otros continentes que en Norteamérica.

Gráfico 2.2 Frecuencia y personas víctimas de violencia física $(\mathrm{n}=229)$

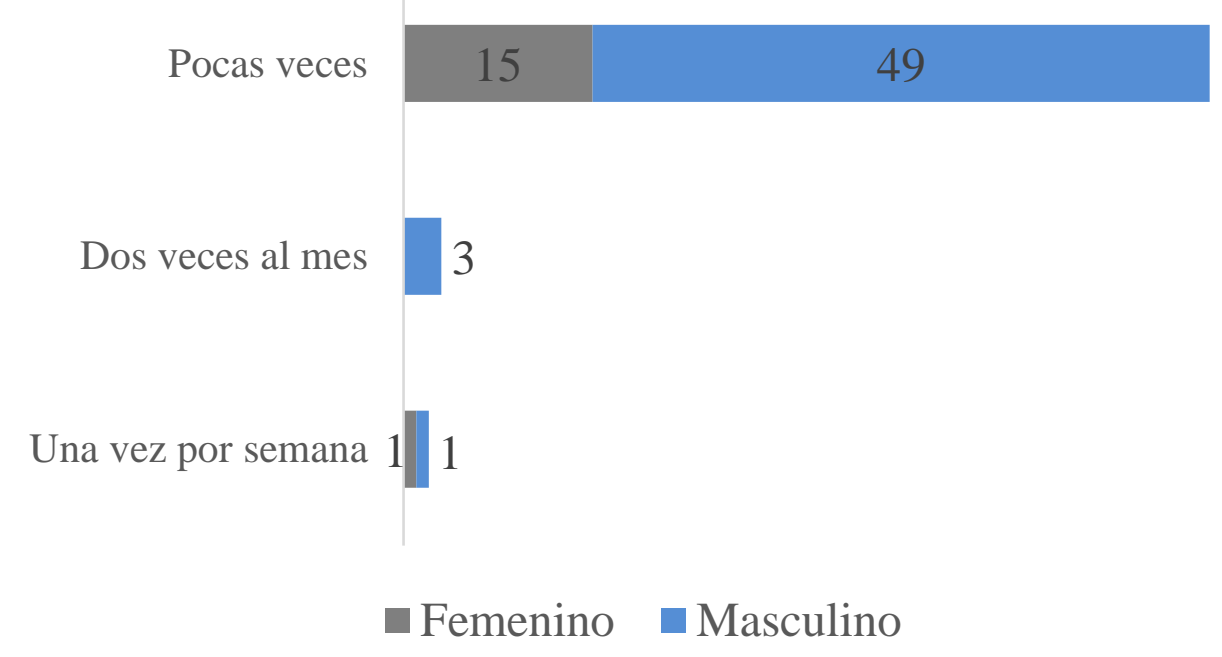

Fuente: Elaboración propia con los datos obtenidos del procesamiento de las respuestas del instrumento

El quinto ítem analizado buscaba conocer la frecuencia con la que habían sido abusados mediante quitar o destruir sus pertenencias, obteniendo que el 17\% al menos en alguna ocasión habían sido víctimas de este tipo de violación, no mostrando diferencia en cuestión de género, solo en cantidad de estudiantes, son más los hombres que reciben este tipo de maltrato. 
El sexto ítem analizado buscaba conocer el porcentaje de presión y la frecuencia frente al consumo de sustancias adictivas, considerando para esta investigación el alcohol, tabaco y otras sustancias adictivas, donde el 7\% de las mujeres sí mencionaron presión para su consumo en pocas ocasiones, este porcentaje para los hombres fue de $3 \%$, en este resultado por cultura se podría pensar que serían los hombres los que con frecuencia se les ofreciera o presionara para ingerirlas, pero el resultado muestra que son las estudiantes quienes reciben más presión.

En el gráfico 2.3 se muestra que el porcentaje de hombres que fueron víctimas de burla en redes sociales es casi el doble de las mujeres que es de $8 \%$, resultado que se muestra fuera de lo esperado por las investigadoras.

Gráfico 2.3 Víctimas de burla en redes sociales ( $\mathrm{n}=229)$

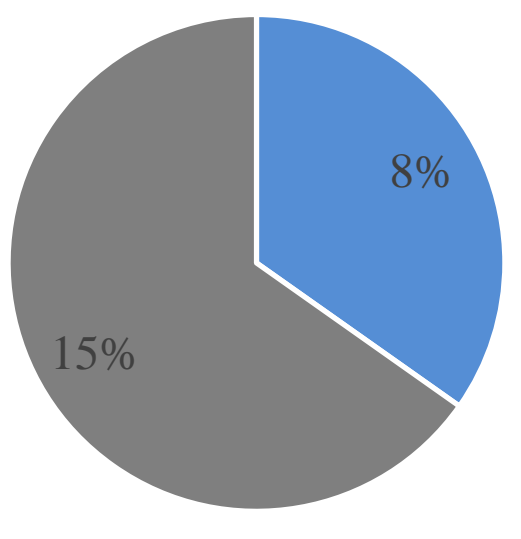

\section{- Femenino $\quad$ Masculino}

Fuente: Elaboración propia con los datos obtenidos del procesamiento de las respuestas del instrumento

Los demás ítems que presentaron información pertinente para esta investigación arrojan datos como que el $2 \%$ de las mujeres y $5 \%$ de los hombres aceptaron vivir violencia familiar, el $15 \%$ de las mujeres fueron víctimas de violencia por su pareja, también el $8 \%$ de los hombres, pero en pocas ocasiones, a pesar que socialmente no lo reconocían por los parámetros o normas sociales.

De los resultados encontrados, el $8 \%$ de las mujeres manifestaba haber sido víctima de violencia mediante redes sociales, en este mismo ítem el resultado de los hombres se detectó un incremento a 15\%, lo que lamentablemente expone una práctica más común en estudiantes adolescentes de nivel básico y medio superior, pero que permanece presente a nivel universitario.

Al $18 \%$ de las mujeres las obligan a hacer cosas que no querían hacer, porcentaje que se ve disminuido en el género masculino con $7 \%$, lo cual muestra que en este rubro sí existe una diferencia importante en cuestión de género y lamentablemente el $44 \%$ de las mujeres y el $39 \%$ de los hombres había sido víctima de robo por algún compañero abusador.

Por último, en el instrumento se les pidió a los estudiantes que indicaran en los últimos doce meses de quiénes habían sentido ser violentados los resultados arrojados fueron alarmantes y se presentan en el gráfico 4.

Las estudiantes manifestaron que el $43 \%$ se sintieron violentadas por sus padres, mientras que en los estudiantes este porcentaje varía de $44 \%$ por sus padres y un $46 \%$ de sus madres, en cuanto al trato que recibieron de sus maestros, las chicas revelaron sentirse victimadas un 33\% por los maestros y un $25 \%$ por las maestras, mientras que los varones el porcentaje en ambos casos fue de $32 \%$. En cuanto a las personas de autoridad o poder con las que conviven, pudiendo ser de la institución educativa, prácticas, servicio social y trabajo los porcentajes revelaron que las mujeres exteriorizaron que el $26 \%$ habían percibido ofensas o maltrato por hombres y el $20 \%$ por autoridades de género femenino, mientras que los hombres notificaron ser violentados el $25 \%$ por su mismo género y el $22 \%$ por el género femenino. 
Sin lugar a duda la cultura entre los compañeros de clase o trabajo presenta los peores resultados, donde las mujeres coinciden que han sido violentadas el 52\% por sus compañeros de ambos géneros mientras que para los hombres el $56 \%$ por sus compañeros de su mismo género y el $48 \%$ por sus compañeras. Lo que nos lleva como docentes a ver la necesidad de impactar directamente en el ambiente del aula para buscar disminuir estos índices.

Gráfico 2.4 Porcentaje de estudiantes que manifiestan haber sido violentados según persona, $(n=229)$

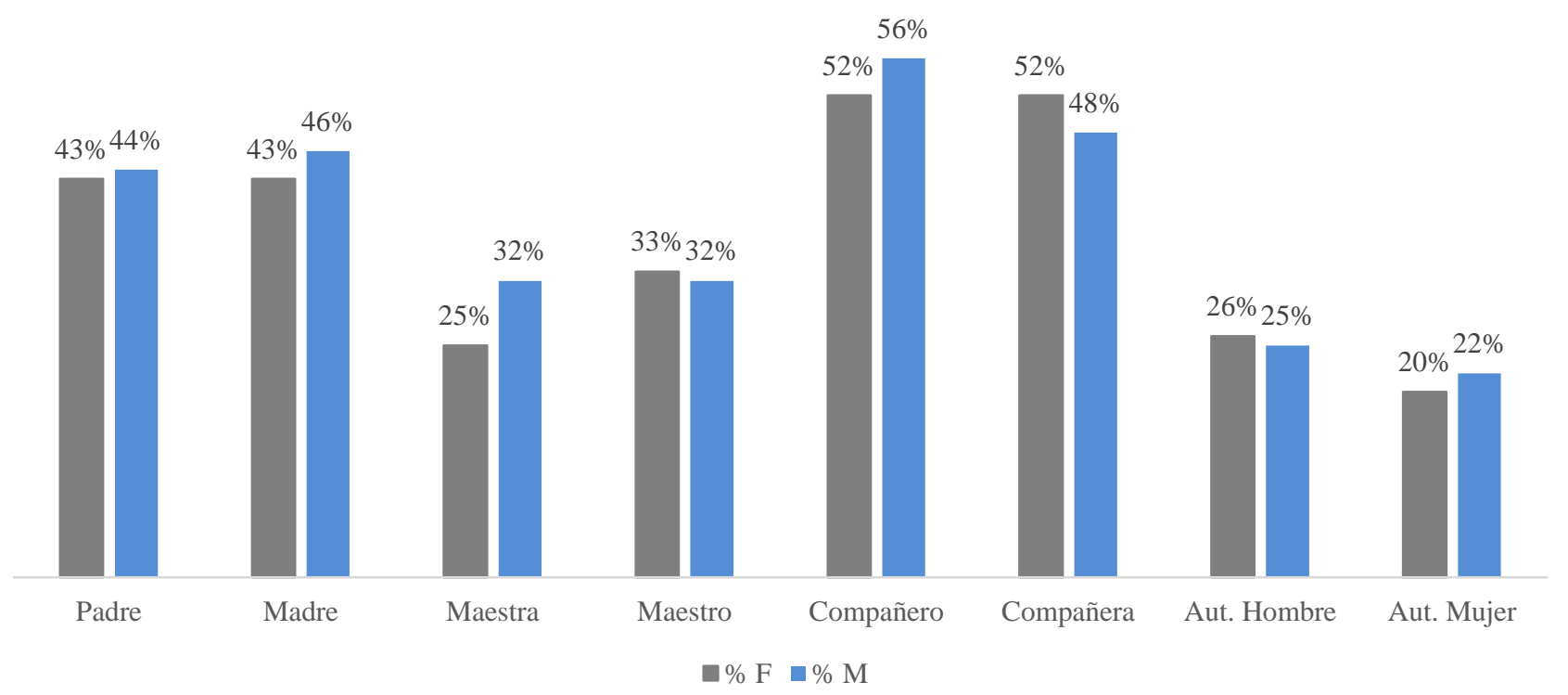

Fuente: Elaboración propia con los datos obtenidos del procesamiento de las respuestas del instrumento

\subsection{Conclusiones}

Esta investigación nos permite demostrar que existe la necesidad de seguir investigando la incidencia de violencia para ambos géneros, no solo centrar el tema hacia el género femenino, pues a de pesar que las mujeres en la mayoría de los ítems presentaban mayor porcentaje de violencia, tres de ellos mostraron que los hombres son sujetos de violencia en mayores índices y que estos parámetros corresponden a los insultos verbales, la violencia física y las burlas en redes sociales, lo que permite concluir que los estudiantes universitarios de género masculino, sí son sujetos de algún tipo de violencia, al igual que las mujeres.

A pesar de que existe una amplia campaña en los medios de comunicación, publicación de propagandas y en instituciones educativas o del estado con protocolos sobre prevención de violencia, estas se dirigen en su mayoría a la violencia sufrida por el género femenino, dejando aún lado al género masculino, incluso pudiendo desmotivar que éstos denuncien tales casos, esto queda refrendado en los resultados aquí plasmados que confirman la presencia de violencia hacia el género masculino, recomendando que es necesario centrar las campañas de prevención de violencia para ambos géneros, invitando a que se denuncie para disminuir o acabar con ella.

La muestra aquí presentada arroja resultados importantes y sustentos enfocados para abordar la violencia desde el ámbito universitario, mas no son suficientes para considerarlos concluyentes, sin embargo, si nos permite orientarnos, para conocer los aspectos a observar en investigaciones subsecuentes y realizar propuestas que promuevan el cambio en la sociedad universitaria en la que vivimos y que estas impacten a mediano plazo en los entornos de los estudiantes.

Promoviendo el respeto y tolerancia, entre los compañeros de clase, hacer énfasis en las acciones que pueden ser consideradas como violencia, las cuales van desde bromas hirientes, ignorar, ridiculizar, intimidar entre muchas otras, así como el uso de redes sociales para molestar de manera temporal o permanente a sus compañeros de clase o trabajo. 
Entre las expresiones de violencia que son referidas por diversos estudios en universitarios incluyen, gritos, discusiones, golpes, amenazas verbales, manifestadas por ambos géneros y en mayor proporción por mujeres, por lo que, los resultados encontrados en este trabajo, reflejan que, existe concordancia con otros estudios realizados a nivel universitario, existe violencia de género, siendo una problemática importante, que requiere implementar una estrategia educativa, para educar en contra de esta realidad. La labor de las instituciones educativas es establecer protocolos para identificar los casos de violencia, brindar atención y seguimiento, dar continuidad y establecer líneas de trabajo que permitan erradicarla, sin importar géneros, logrando espacios académicos libres de violencia, con equidad e igualdad de género. También considerar presentar un proyecto para disminuir o erradicar la violencia a través de las redes sociales, desde el programa de inducción alfabetizando o a través del docente dentro de su cátedra realizar una campaña de no violencia entre los estudiantes a través de las redes sociales, como lo proponen Latorre, Tena, Murillo y Toscano, (2020)

\subsection{Referencias}

Águila, Y, Hernández, V. y Hernández, V. (2016). Las consecuencias de la violencia de género para la salud y formación de los adolescentes Rev.Med.Electrón. vol.38 no.5 Matanzas sep.-oct. 2016. versión On-line ISSN 1684-1824. http://scielo.sld.cu/scielo.php?script=sci_arttext\&pid=S168418242016000500005

Bueno, M. (2010). Hombres maltratados, una realidad silenciada. [Recuperado el 26 de octubre de 2016]. Disponible en: http://www.mateobuenoabogado.com/blog/hombres-maltratados-una-realidadsilenciada/?print=pdf

Buvinic M. (2005). Violencia, crimen y desarrollo social en América Latina y el Caribe. Papeles de Población, 11, 167 - 214. Recuperado en octubre de 2016, de Redalyc.org: http://www.redalyc.org/articulo.oa?id=11204309

Dobarro, A., García, D. Á., \& Núñez, J. C. (2014). Cuve3: Instrumentos para evaluar la violencia escolar. International Journal of Developmental and Educational Psychology (Revista INFAD de Psicología)., 5 (1), 487-492. https://www.redalyc.org/articulo.oa?id=349851788055

Ehrenfeld L., Noemí. 2016. "Las violencias y los jóvenes universitarios, una experiencia para reflexionar”. Trabajo Social 18: 93-110. Bogotá: Departamento de Trabajo Social, Facultad de Ciencias Humanas, Universidad Nacional de Colombia.

Giraldo, Octavio. (1972). El machismo como fenómeno psicocultural. Revista Latinoamericana de Psicología, Vol. 4, No 3, pp. 295-309.

Guedes, A. C., Bott, S., García-Moreno, C., \& Colombini, M. (2017). Cerrar la brecha: revisión mundial acerca de las intersecciones entre la violencia contra la mujer y la violencia contra los niños y las niñas. https://repositorio.unal.edu.co/bitstream/handle/unal/64831/58576-296661-1-

SM.pdf? sequence $=1 \&$ is Allowed $=\mathrm{y}$

Ibanez, D. (2017). La violencia de género en Ecuador: un estudio sobre los universitarios. Rev. Estudos Feministas. Vol. 25, No. 3, pp. 1313-1327. ISSN 0104-026X. http://dx.doi.org/10.1590/18069584.2017v25n3p1313.

Índice global de Paz (2018). https://www.esglobal.org/indice-paz-global-2018/

INEGI (2018). https://www.inegi.org.mx/temas/victimizacion/

INEGI

https://www.inegi.org.mx/contenidos/saladeprensa/aproposito/2019/Violencia2019_Chi.pdf

(2019).

Latorre, M. L. Á., Tena, R. O., Murillo, D. A. F., \& Toscano, M. Ú. P. (2020). El rendimiento académico en estudiantes universitarios y su relación con la percepción del clima escolar. Prácticas y reflexiones sobre la educación contemporánea, 99 
Ley General de acceso de las mujeres a una vida libre de violencia http://www.diputados.gob.mx/LeyesBiblio/pdf/LGAMVLV_130418.pdf

Mateo, V. F., Mesas, C. G., \& Ruiz, I. M. (2015). Violencia entre iguales: Resultados de un estudio descriptivo de la provincia de Valencia. Información Psicológica, (94), 36-48.

OMS. (2002). Informe mundial sobre la violencia y la salud. Sinópsis, Ginebra. https://www.who.int/violence_injury_prevention/violence/world_report/en/abstract_es.pdf

Orellana, R. Vigil, Á. y Arrebola, A. (2020). Los estereotipos de género y las actitudes sexistas de los estudiantes universitarios en un contexto multicultural. Profesorado, Revista de Currículum y Formación del Profesorado, 24(1), 284-303.

Organización Mundial de la Salud. (2018). https://www.who.int/topics/gender/es/ consultado el 02 de abril 2020

Positive peace report (2018). http://visionofhumanity.org/app/uploads/2018/11/Positive-Peace-Report2018.pdf

Safranoff, A. (2017). ¿Qué significa ser maltratada? El estudio de la violencia de género a través de datos de encuesta. Revista Latinoamericana de Metodología de las Ciencias Sociales, 7 (1), e019. https://doi.org/10.24215/18537863e019

Sanmartín, J. (2007) ¿Qué es violencia? Una aproximación al concepto y a la clasificación de la violencia. $\Delta \alpha i^{\prime} \mu \omega v$. Revista de Filosofía, $n^{\circ} 42,2007,9-21$ ISSN: 1130-0507

Torres, M. (2015). Mujeres y Derechos Humanos ante la violencia de género. Cahiers de civilisation espagnole contemporaine (En ligne), 15|2015, mis en ligne le 19 janvier 2016, consulté le 28 avril 2020. URL: http://journals.openedition.org/ccec/6000; DOI: https://doi.org/10.4000/ccec.600

UNESCO (2019) Behind the numbers: ending school violence and bullying. https://unesdoc.unesco.org/ark:/48223/pf0000366483 